\title{
A influência da atividade física no sono e desempenho acadêmico
}

\author{
The influence of physical activity on sleep and academic performance
}

La influencia de la actividad física en el sueño y el rendimiento académico

Lucas Nogueira Diniz ${ }^{1 *}$, Felipe Ferreira Lima1, Lorena de Souza Carvalho', Paulo Arthur Mendes Milhomem¹, Pedro Henrique Omena Celestino¹, Gustavo Mariano Rodrigues Santos ${ }^{1}$, Pedro Felipe Pires Simplício', Tatiana Teixeira Barral de Lacerda¹.

\section{RESUMO}

Objetivo: Evidenciar se a prática de atividade física e a qualidade do sono influenciam no desempenho acadêmico de estudantes de ensino médio de uma escola privada de Belo Horizonte. Métodos: Trata-se de um estudo observacional descritivo, realizado com 33 adolescentes de 15-17 anos. Foram aplicados questionários relacionados à qualidade do sono (Escala de Sonolência de Epworth), frequência/intensidade de atividades físicas (Questionário internacional de Atividades Físicas) e um questionário de identificação produzido pelos autores. Essas informações foram comparadas ao desempenho acadêmico dos alunos, mensurados por meio de notas, e avaliadas usando testes estatísticos apropriados. Resultados: Os resultados não evidenciaram influência da prática de atividade física e da qualidade do sono no desempenho acadêmico dos estudantes avaliados. Ainda que a pesquisa não tenha conseguido evidenciar relações diretas, diversos estudos que abordam o tema foram encontrados e sustentam a hipótese de que as relações sugeridas sejam pertinentes. Conclusão: Os resultados do presente estudo não confirmam os achados encontrados na literatura de uma associação entre atividades físicas, sono e desempenho acadêmico. Acredita-se que o tamanho limitado da amostra possa ter influenciado os resultados.

Palavras-chave: Atividade motora, Desempenho acadêmico, Sono, Aprendizagem.

\begin{abstract}
Objective: To evidence the existence of a positive relationship among physical activity, sleep quality and academic performance in high school students of a private school at Belo Horizonte. Methods: It's a descriptive observational study, carried out with 33 adolescents aged 15-17 years. Questionnaires related to sleep quality (Epworth Sleepiness Scale), frequency / intensity of physical activities (International Physical Activity Questionnaire) and an identification questionnaire produced by the authors were applied. This results was compared to students grades through Pearson's Correlation Analysis. Results: It was not possible to establish relationships with statistical significance in the study. Even if the research did not succeed to show direct relacions about the variables, there is so many studies that proves and supports the hypothesis that exist a strong association about them. Conclusion: The results of the present study don't confirm the literature conclusions of the relationship among motor activity, sleep and academic performance. We believe that this fact is due a limited sample size of our study.
\end{abstract}

Keywords: Motor activity, Academic performance, Sleep, Learning.

${ }^{1}$ Pontifícia Universidade Católica de Minas Gerais (PUC Minas), Belo Horizonte - MG.

*E-mail: lucasdiniz95@hotmail.com

SUBMETIDO EM: 8/2020

ACEITO EM: 9/2020

PUBLICADO EM: 10/2020 


\section{RESUMEN}

Objetivo: Evidenciar si la actividad física y la calidad del sueño influyen en el rendimiento académico de los estudiantes de secundaria, en una escuela privada en Belo Horizonte. Métodos: Estudio observacional descriptivo, realizado con 33 adolescentes de 15 a 17 años. Se aplicaron cuestionarios relacionados con la calidad del sueño (Escala de somnolencia de Epworth), la frecuencia / intensidad de las actividades físicas (Cuestionario internacional de actividad física) y un cuestionario de identificación creado por los autores. Esta información se comparó con el rendimiento académico de los estudiantes, se midió usando calificaciones y se evaluó usando pruebas estadísticas apropiadas. Resultados: Los resultados no mostraron ninguna influencia de la actividad física y la calidad del sueño en el rendimiento académico de los estudiantes evaluados. Aunque la investigación no ha logrado mostrar relaciones directas, se han encontrado varios estudios que abordan el tema y respaldan la hipótesis de que las relaciones sugeridas son relevantes. Conclusión: Los resultados del presente estudio no confirman los hallazgos encontrados en la literatura de una asociación entre actividades físicas, sueño y rendimiento académico. Se cree que el tamaño limitado de la muestra puede haber influido en los resultados.

Palabras clave: Actividad motora, Rendimiento académico, Sueño, Aprendizaje.

\section{INTRODUÇÃO}

A supervalorização do conhecimento e da formação acadêmica faz com que as pessoas busquem estudar cada vez mais com o intuito de crescer no âmbito profissional. Atrelada a essa procura pelo desenvolvimento intelectual nota-se um aumento da prevalência de diversas doenças psíquicas. $O$ estresse vem sendo reconhecido como importante influenciador no desempenho profissional, acadêmico e na saúde dos indivíduos. Muitas vezes, o período que antecede o ingresso na universidade é reconhecido como um momento causador de ansiedade, estresse e até depressão. As causas relacionadas a tais desconfortos nesse período são diversas, como, por exemplo, a pressão para o sucesso no exame, a interferência familiar e a concorrência. Essas condições, por sua vez, além de prejudicar a qualidade de vida, interferem negativamente no processo de assimilação de conhecimentos (SANTOS FS, et al., 2017).

Em adolescentes e adultos jovens, esses fatores se tornam mais relevantes por ser um período em que surgem maiores responsabilidades, novas perspectivas pessoais e profissionais. Tudo isso está atrelado à inexperiência e às alterações físicas e mentais do indivíduo. Diante do elevado número de tarefas a serem realizadas, a principal consequência é a diminuição da quantidade e da qualidade do sono.

Em um estudo com 616 adolescentes, de ambos os sexos, em uma faixa etária entre 10 e 19 anos, foi constatada uma prevalência de baixa duração de sono de 53,6\% (FELDEN EPG, et al., 2016). O sono suficiente é essencial para uma saúde ideal - alguns dos inúmeros processos que ocorrem durante o sono incluem consolidação da memória, liberação de metabólitos cerebrais e restauração dos sistemas nervoso, imunológico, esquelético e muscular (KLINE CE, 2014).

A atividade física (AF) aparece como uma intervenção para melhora da qualidade do sono e, consequentemente, facilitação do processo de aprendizagem. Apesar da existência de poucas pesquisas experimentais envolvendo pacientes com distúrbios do sono significativos, as evidências disponíveis sugerem que o exercício é promissor como uma terapia não farmacológica (KLINE CE, 2014).

Em um estudo com 463 alunos da $7^{\mathrm{a}}$ série que foram acompanhados durante 2 anos, a participação em $\mathrm{AF}$ foi positivamente associada à melhora do desempenho acadêmico desde o início até o acompanhamento final da pesquisa. Além disso, a participação em AF que requerem habilidades motoras mais complexas e AF individuais foi diretamente associada à melhora do desempenho acadêmico (ISHIHARA T, et al., 2020).

As atividades físicas proporcionam uma melhora nas funções cognitivas e, por isso, facilitam um bom desempenho acadêmico em crianças e adolescentes. Isso ocorre, por meio de um trabalho de ação motora 
e psíquica que são atraídos pelo exercício, o que contribui com o processo de aprendizagem. Ao promover a aptidão física e a saúde metabólica, o exercício físico pode favorecer uma melhora de funções cognitivas específicas.

Entre os benefícios cognitivos de um estilo de vida ativo, parece que o exercício físico pode beneficiar especificamente as funções executivas, que compreendem controle inibitório, planejamento, memória operacional, tomada de decisão e flexibilidade cognitiva. Essas funções são necessárias para o desempenho das atividades diárias, sendo particularmente importante para o desenvolvimento cognitivo e motor (MACHADO JM, 2017).

Para chegarmos a resultados expressivos, a prática de exercícios deve ser feita de forma contínua e em intensidade adequada. A recomendação atual é que todo jovem pratique 60 minutos ou mais de AF, moderadas ou vigorosas, diariamente (SOCIEDADE BRASILEIRA DE PEDIATRIA, 2017). Entretanto, atualmente, uma aprendizagem em alto nível e bons resultados em avaliações educacionais tem sido os principais objetivos de pais e alunos.

Com isso, priorizam longos períodos dedicados aos estudos e ignoram a prática de AF no tempo livre. Todavia, praticar alguma AF e/ou esporte nessa fase da vida não traz prejuízo ao aprendizado escolar e o tempo dedicado à educação dentro da escola deveria respeitar o tempo para a participação em AF (DIAMOND A e LEE K, 2011).

Nesse sentido, partindo do pressuposto que um indivíduo saudável possui um desempenho cognitivo superior e que a prática de AF contribui para a manutenção da sua saúde, o presente estudo tem como objetivo sustentar a hipótese de que a prática de AF influencia positivamente no desempenho acadêmico e na qualidade do sono de jovens.

Dessa maneira, tendo em vista o fato de que o exercício físico é uma atividade que, na grande maioria das vezes, não requer gastos financeiros, a sustentação dessa hipótese traria grandes benefícios populacionais. Isso ocorreria por ser uma intervenção acessível a todos. A sua elucidação e divulgação permitirá que jovens e indivíduos em geral, das mais diversas classes sociais, melhorem o seu desempenho escolar e atinjam seus objetivos com a ajuda da atividade física regular.

\section{MÉTODOS}

Para confirmar a hipótese de que a AF e o sono exercem uma influência positiva sobre o desempenho acadêmico foi realizado um estudo observacional descritivo. Os dados foram obtidos por meio de questionários aplicados pelos próprios pesquisadores. A coleta de dados foi realizada em uma escola particular na cidade de Belo Horizonte - MG.

O questionário foi entregue aos 150 alunos regularmente matriculados no segundo ano do ensino médio. Foram incluídos no estudo todos aqueles que concordaram em participar, bem como seus respectivos responsáveis legais, e que devolveram os questionários preenchidos até a data estipulada pelos autores. Não houve critérios de exclusão

O primeiro questionário foi confeccionado pelos autores e teve como objetivo conhecer os entrevistados (Questionário de identificação dos participantes - Dados Suplementar). Além disso, o instrumento foi criado com o intuito de compreender o contexto em que os entrevistados estavam inseridos, bem como fatos importantes da sua rotina que poderiam interferir na pesquisa.

Posteriormente, para avaliar a frequência e a intensidade das atividades físicas realizadas pelos avaliados, foi utilizada a Escala Internacional de Atividade Física IPAC - Versão curta (Dados Suplementar). Esse questionário foi criado em 1998 pela Organização Mundial da Saúde (OMS) com o intuito de estimar o nível de prática habitual de atividade física de populações de diferentes países (GUEDES DP, et al., 2005).

Essa ferramenta possui 4 perguntas com 2 itens cada e busca quantificar o número médio de dias da semana que o indivíduo praticou uma determinada atividade, quanto tempo foi gasto nessa atividade e a sua intensidade. 
A validade e reprodutibilidade do IPAQ versão curta em adolescentes, versão utilizada no atual estudo e população semelhante, foi verificada através de um estudo com uma amostra de 161 adolescentes de 3 escolas de Londrina, Paraná. Como resultado, o artigo nos traz que o instrumento apresenta propriedades aceitáveis na monitorização de atividade física para a população acima de 14 anos (GUEDES DP, et al., 2005).

Para avaliar a sonolência diurna e, consequentemente, a qualidade do sono dos entrevistados, foi utilizada a Escala de Sonolência de Epworth (Dados Suplementar). Essa ferramenta sugere algumas situações e os indivíduos devem responder com nota de 0 a 3, sendo que: 0 - Nenhuma chance de cochilar; 1 - Pequena chance de cochilar; 2 - Moderada chance de cochilar e 3 - Alta chance de cochilar. Ao final as notas são somadas e classifica-se como sono normal se o resultado foi de 1 a 6 pontos, média sonolência se de 7 ou 8 pontos e sonolência anormal para uma pontuação entre 9 e 24 .

O desenvolvimento dessa escala para utilização no Brasil (versão em português) foi feito através das etapas de: tradução; retrotradução; comparação entre a tradução e a retrotradução (por um comitê); e aplicação em indivíduos bilíngues. Para sua validação, ela foi aplicada em indivíduos de 18 a 65 anos, com uma amostra total de 114 pacientes e 21 controles incluídos. Os resultados mostraram que o ESSE-BR é um instrumento válido e confiável para a avaliação da sonolência diurna e equivalente a sua versão original (BERTOLAZI, et al., 2009).

Os resultados encontrados nos questionários anteriormente citados foram comparados com a média global das notas dos estudantes. Apesar de reconhecer que as notas não são o meio mais correto de se mensurar a capacidade cognitiva de um indivíduo, já que existem habilidades e competências que não são avaliadas em provas convencionais, entende-se que, para o estudo, elas seriam a forma mais objetiva e razoável de se fazer uma análise.

Os dados obtidos foram tabulados no programa estatístico Statistical Package for the Social Sciences (SPSS/PC) versão 17.0. Inicialmente foi realizada análise descritiva dos dados e em seguida, investigou-se a existência de diferenças significativas, através da ANOVA, entre o desempenho acadêmico e a prática de atividade física e entre o desempenho acadêmico e a qualidade do sono.

O teste $t$ de student foi realizado para verificar diferença entre a média de notas e a prática ou não de atividade física. Também foi utilizado o teste Qui-quadrado para avaliar se há diferença entre sexo, prática de atividade física e mudança de hábitos de atividade física em relação ao sono. Foi considerado um nível de significância de $\mathrm{p}=0.05$.

Vale ressaltar que todos os participantes assinaram um termo de assentimento em participar da pesquisa e seus responsáveis assinaram um termo de consentimento. O projeto foi aprovado pelo Comitê de Ética em pesquisa da PUC Minas, sob parecer número: 2.428.620.

\section{RESULTADOS}

Dos 150 questionários entregues, apenas 33 foram retornados, apesar das diversas estratégias e tentativas realizadas pelos autores. Assim, foram avaliados 33 sujeitos da segunda série do ensino médio, sendo $81,8 \%$ do sexo feminino.

Em relação a idade, a maior parte (87.9\%) tem 16 anos, 3\% tem 15 anos e 9,1\%, 17 anos. A maioria dos adolescentes reside com os pais (66.7\%); $27.3 \%$ deles com um dos pais e apenas $6 \%$ com nenhum dos pais. A caracterização dos adolescentes sobre a prática de atividade física está descrita na Tabela 1.

Com o Questionário de Identificação dos Participantes, dentre outras informações, foi possível constatar que $33.4 \%$ não praticavam qualquer tipo de atividade física. Os valores referentes à qualidade do sono dos estudantes, através da Escala de Sonolência de Epworth, pode ser visto na Tabela 2. 
Tabela 1 - Caracterização dos estudantes quanto ao padrão de atividade física.

\begin{tabular}{lc}
\hline Variáveis & № Casos (\% Frequência) \\
\hline Prática de atividade física e frequência & \\
\hline Não pratica & $11(33.4 \%)$ \\
Sim, 2x por semana & $7(21.2 \%)$ \\
Sim, 3 ou 4x por semana & $8(24.2 \%)$ \\
Sim, 5x ou mais por semana & $7(21.2 \%)$ \\
\hline Tempo que mantém a rotina & \\
\hline 1 mês & $6(18.2 \%)$ \\
6 meses & $7(21.2 \%)$ \\
12 meses & $4(12.1 \%)$ \\
24 meses & $16(48.5 \%)$ \\
\hline Mudança de hábitos de atividade física & \\
\hline Sim & $13(39.4 \%)$ \\
Não & $20(60.6 \%)$ \\
\hline Mudança de hábitos do sono & \\
\hline Sim & $13(39.4 \%)$ \\
Não & $20(60.6 \%)$ \\
\hline A rotina da últimas semanas condiz com a última do ano \\
\hline Sim & $20(60.6 \%)$ \\
Não & $13(39.4 \%)$ \\
\hline
\end{tabular}

Fonte: Diniz LN, et al., 2018.

Tabela 2 - Qualidade do sono.

\begin{tabular}{ll}
\hline Variáveis & № Casos (\% Frequência) \\
\hline Sono normal & $8(24.2 \%)$ \\
Média de sonolência & $5(15.2 \%)$ \\
Sonolência anormal & $20(60.6 \%)$ \\
\hline
\end{tabular}

Fonte: Diniz LN, et al., 2018.

Constata-se, portanto, que mais da metade dos entrevistados (60,6\%) apresentavam sonolência anormal, $15.2 \%$ apresentavam média sonolência e que apenas $24,2 \%$ apresentavam uma sonolência considerada normal. Vale ressaltar que uma das perguntas do instrumento avalia a chance de o entrevistado cochilar sentado e lendo, atividade de grande importância para um estudante do ensino médio.

Os resultados encontrados neste tópico foram: $24,24 \%$ nenhuma chance de cochilar, $24,24 \%$ pequena chance de cochilar, $27,27 \%$ moderada chance de cochilar e $24,24 \%$ alta chance de cochilar. Constata-se, portanto, que $75,75 \%$ dos entrevistados apresentavam pelo menos alguma chance de cochilar estando sentados e lendo, o que é um dado interessante.

A Tabela 3 evidencia o comportamento dos estudantes em relação à prática de atividade física avaliada através do Questionário internacional de atividade física.

Tabela 3 - Prática de Atividade Física.

\begin{tabular}{ll}
\hline Variáveis & № Casos (\% Frequência) \\
\hline Inativo & $10(30.3 \%)$ \\
Minimamente ativo & $6(18.2 \%)$ \\
Ativo & $17(51.5 \%)$ \\
\hline
\end{tabular}

Fonte: Diniz LN, et al., 2018. 
Os resultados evidenciam que a maioria dos entrevistados $(51,5 \%)$ pode ser considerado ativo ao se analisar a frequência e a intensidade das atividades físicas praticadas. Por outro lado, $30.3 \%$ dos participantes foram considerados inativo e $18.2 \%$ minimamente ativos. Nesse sentido, constata-se uma elevada quantidade de jovens que não se exercitam ou que o fazem em intensidade e frequência inadequadas. Os resultados da ANOVA entre o desempenho acadêmico, mensurado pela média de notas dos alunos, e a prática de atividade física não apresentou diferença estatisticamente significativa $(p=0.955)$, bem como também não foi observada diferença entre o desempenho acadêmico e a qualidade do sono $(p=0.201)$.

Ao realizar o teste t student não foi encontrado diferença estatisticamente significativa entre a média de notas e a prática ou não de atividade física (p:0.295). Não foi possível observar diferenças significativas quanto ao sexo, prática de atividade física e mudança de hábitos de atividade física naqueles com sono normal ou não (Tabela 4).

Tabela 4 - Qualidade do Sono.

\begin{tabular}{|c|c|c|c|}
\hline \multirow{2}{*}{ Variáveis } & \multicolumn{2}{|c|}{ Qualidade do sono } & \multirow{2}{*}{$\begin{array}{l}\text { Valor } \\
\text { de } p\end{array}$} \\
\hline & Sono normal & Sonolência média ou anormal & \\
\hline \multicolumn{4}{|c|}{$\mathrm{CH}_{\mathrm{N}}$} \\
\hline Feminino & $6(22.2 \%)$ & $21(77.8 \%)$ & \multirow{2}{*}{0,456} \\
\hline Masculino & $2(33.3 \%)$ & $4(66.7 \%)$ & \\
\hline \multicolumn{4}{|c|}{ Atividade física } \\
\hline Inativo & $3(30.0 \%)$ & $7(70.0 \%)$ & \multirow{2}{*}{0,461} \\
\hline Ativo & $5(21.7 \%)$ & $18(78.3 \%)$ & \\
\hline \multicolumn{4}{|c|}{ Mudança hábitos atividade física } \\
\hline Sim & $3(23.1 \%)$ & $10(76.9 \%)$ & \multirow{2}{*}{0,619} \\
\hline Não & $5(25.0 \%)$ & $15(75.0 \%)$ & \\
\hline
\end{tabular}

Fonte: Diniz LN, et al., 2018.

\section{DISCUSSÃO}

Os resultados deste estudo analítico observacional procuraram avaliar a relação entre o perfil de atividade física (AF), a percepção da qualidade do sono e o desempenho acadêmico (DA) em alunos do $2^{\circ}$ ano do ensino médio. Apesar de não ter sido possível encontrar uma correlação estatisticamente relevante, algumas análises interessantes podem ser feitas ao observar dados fornecidos pela pesquisa e compará-los com outros estudos relacionados ao tema. Nesse sentido, vale ressaltar que outros trabalhos nacionais e internacionais, que também buscaram estabelecer uma conexão entre as variáveis $A F, D A$ e qualidade do sono, obtiveram êxito. (SANTANA CCA, et al., 2020) (PARUTHI S, et al., 2016) (HYSIN M, et al., 2016).

Acreditamos que a insignificância estatística desta pesquisa se deva ao tamanho da amostra. Uma das limitações deste trabalho foi não considerar o fato de que, ao trabalhar com um público menor de 18 anos, haveria um impacto significativo na adesão ao estudo, seja por problemas com Termo de consentimento livre e esclarecido (TCLE) ou ausência de estímulo para participação. Dessa forma, a amostra obtida foi extremamente reduzida (22\% do previsto inicialmente), o que restringiu a análise dos dados e consequentemente interferiu negativamente no estabelecimento de uma relação concreta. Entretanto, toda a literatura pesquisada sustenta a hipótese que a AF age como efeito potencializador do DA e de melhora da qualidade do sono.

Um exemplo é uma revisão sistemática que incluiu 45 estudos em jovens menores de 20 anos e concluiu que a relação sugerida é plausível. Esse estudo concluiu que as evidências são robustas no sentido haver uma relação positiva entre AF e DA embasando-se em pesquisas transversais. Ao analisar estudos longitudinais, uma relação forte foi estabelecida entre aptidão cardiorrespiratória e DA. Essa associação ocorre, ainda segundo o autor, por meio de uma facilitação do processo de angiogênese no córtex motor causada pela prática de atividades físicas, o que aumenta o fluxo sanguíneo e oxigenação dos tecidos cerebrais (SANTANA CCA, et al., 2020). 
Outro estudo procurou estabelecer relações entre diversas variáveis relacionadas ao tema, como AF, cognição, aprendizagem, função cerebral e concentração. Trata-se de uma revisão sistemática que incluiu 137 trabalhos e analisou apenas jovens entre 5-13 anos. Os autores concluem que existem evidências suficientes para sugerir que uma relação entre todas essas variáveis seja estabelecida. Entretanto, afirmam que não foi possível correlacionar de forma direta cada variável com a sua exata contribuição no processo e que, por isso, novos estudos devam ser realizados (DONNELLY JE, et al., 2016).

Neste sentido, é orientado que jovens entre 5 e 17 anos pratiquem de ao menos 60 minutos de atividade física de intensidade moderada a vigorosa diariamente. Além disso, salienta-se que acima deste valor temporal há benefício adicional de saúde (WORLD HEALTH ORGANIZATION, 2011).

No presente estudo, foi possível observar que apenas de $21 \%$ da amostra realizava AF de acordo com a recomendação da OMS e que 33,4\% não praticavam qualquer AF. Trata-se de um percentual extremamente preocupante, uma vez que a AF contribui em diversos aspectos para uma melhor qualidade de vida.

Um estudo com 11.110 jovens europeus de 14 a 16 anos buscou relacionar AF e participação em esportes com bem estar, ansiedade e sintomas depressivos. Os autores concluíram que a frequência de atividade praticada foi positivamente correlacionada com o bem-estar e negativamente correlacionada com sintomas de ansiedade e depressão em ambos os sexos. Além disso, apesar da recomendação da prática de 60 minutos diários de AF para jovens, este estudo não encontrou evidências que exijam uma prática diária para que os benefícios na saúde mental sejam percebidos (MCMAHON EM, et al., 2017).

Outro dado interessante observado no presente estudo foi que $60,6 \%$ do total da amostra apresentava sonolência anormalmente presente. Trata-se de um percentual próximo ao encontrado por FELDEN EPG, et al., 2016, que foi de $53,6 \%$ de baixa duração do sono. O estudo realizado por esse autor analisou 516 adolescentes entre 10 e 19 anos e buscou estabelecer relações entre comportamento sedentário e variáveis de sono (sonolência diurna e duração de sono). Os resultados mostraram que os adolescentes com baixa duração do sono apresentaram maior sonolência diurna $(p<0.001)$ e maior sonolência em sala de aula $(p<$ 0.001). Além disso, aqueles com maior sonolência tinham 4.97 (IC: $2.50-9.87$ ) vezes mais chance de ter comportamento sedentário elevado.

Em concordância com os resultados encontrados, a Academia Americana de Medicina do Sono publicou em 2016 um consenso com recomendações relacionadas à qualidade do sono em diversas faixas etárias. Segundo o documento, que foi produzido a partir de uma revisão sistemática com 864 artigos, o ideal é que jovens de 13 a 18 anos tenham uma noite de sono entre 8 e 10 horas em 24 horas, de forma regular.

Ainda segundo o consenso, dormir o tempo recomendado está associado a melhora na atenção, aprendizado, comportamento, memória, estabilidade emocional, qualidade de vida e saúde física e mental. Por fim, conclui que o não seguimento das recomendações está relacionado a problemas de comportamento, atenção a aprendizado, além de facilitar o aparecimento de sintomas depressivos e suicidas (PARUTHI S, et al., 2016).

Fica evidente, então, a importância de um sono de qualidade para a saúde de um indivíduo jovem. Mas também é possível relacioná-lo diretamente a uma melhora da performance acadêmica na faixa etária em análise. Um estudo comparou as notas de 7798 adolescentes com seus hábitos de sono (duração, eficiência, déficit, e diferenças entre o "tempo de cama" nos dias de semana e finais de semana).

A maioria desses parâmetros foi associado com uma piora do DA, sendo que a baixa duração e o déficit de sono mostraram-se os mais importantes. Vale ressaltar que as melhores médias de notas foram encontradas nos indivíduos que possuíam uma duração do sono de 7-9 horas e que um tempo maior do que esse não mostrou benefício no DA, mas sim uma leve piora (HYSIN M, et al., 2016).

\section{CONCLUSÃO}

Os resultados do presente estudo não confirmam os achados encontrados na literatura de uma associação entre atividades físicas, sono e desempenho acadêmico. Também não foram encontradas diferenças em 
relação ao sexo. Considera-se necessária e relevante a realização de novos estudos, pois apesar de haver na literatura uma base consolidada sobre o tema, um tamanho limitado da amostra deste estudo pode ter influenciado os resultados, não permitindo inferir uma análise de associação com significância estatística.

\section{AGRADECIMENTOS E FINANCIAMENTO}

Agradecemos à Pontifícia Universidade Católica de Minas Gerais (PUC Minas), nossa Alma Mater por toda contribuição e capacitação dos autores, proporcionando um ambiente favorável à pesquisa e fomentando a busca incessante pelo saber, além da supervisão e auxílio de nossa orientadora. Destaca-se o financiamento feito pelo Programa de Bolsas de Iniciação Científica e Tecnológica Institucional da FAPEMIG - PROBIC, número de concessão: 2018/1653.

\section{REFERÊNCIAS}

1. BERTOLAZI AN, et al. Validação da escala de sonolência de Epworth em português para uso no Brasil. Jornal Brasileiro de Pneumologia, 2009; 35(9): 877-883.

2. DIAMOND A, LEE K. Interventions shown to aid executive function development in children 4 to 12 years old. Science, 2011; 6045(333): 959-64.

3. DONNELLY JE, et al. Physical Activity, Fitness, Cognitive Function, and Academic Achievement in Children. Medicine \& Science in Sports \& Exercise, 2016; 48(6): 1197-1222.

4. FELDEN EPG, et al. Adolescentes com sonolência diurna excessiva passam mais tempo em comportamento sedentário. Rev Bras Med Esporte, 2016; 22(3): 186-190.

5. GUEDES, DP, et al. Reprodutibilidade e validade do Questionário Internacional de Atividade Física em adolescentes. Rev Bras Med Esporte, 2005; 11(2): 151-158.

6. HYSING M, et al. Sleep and academic performance in later adolescence: results from a large population-based study. Journal of Sleep Research, 2016; 25(3): 318-324.

7. ISHIHARA T, et al. Relationship between participation in specific sports and academic performance in adolescents: a two year longitudinal study. Scandinavian Journal of Medicine e Science in Sports, 2020; 30(8): 1471-1482.

8. KLINE CE. The bidirectional relationship between exercise and sleep: implications for exercise adherence and sleep improvement. Am J Lifestyle Med, 2014; 8(6): 375-379.

9. LESSA RT, et al. A privação do sono e suas implicações na saúde humana: uma revisão sistemática da literatura. Revista Eletrônica Acervo Saúde, 2020; 56: 3846.

10. MACHADO JM. Os efeitos da atividade física na aprendizagem e no desenvolvimento motor infantil. Dissertação (Graduação em Educação Física) - Centro Universitário Hermínio da Silveira IBMR - Laureate International Universities. Curso de Educação Física, Rio de Janeiro, 2017; 39p.

11. MCMAHON EM, et al. Physical activity in European adolescents and associations with anxiety, depression and well-being. European Child \& Adolescent Psychiatry, 2016; 26(1): 111-122.

12. OLIVEIRA LMFT, et al. Exercise or physical activity: which is more strongly associated with the perception of sleep quality by adolescents? Revista Paulista de pediatria, 2018; 36(3): 322-328.

13. OLIVEIRA MCS, et al. Qualidade do sono e sonolência excessiva diurna em acadêmicos de medicina. Revista Eletrônica Acervo Saúde, 2020; 12(10): 4011.

14. PARUTHI S, et al. Declaração de consenso da Academia Americana de Medicina do Sono sobre a quantidade recomendada de sono para crianças saudáveis: metodologia e discussão. Journal of Clinical Sleep Medicine, 2016; 12(11): 1550-9397.

15. QIAN J, et al. Ghrelin is impacted by the endogenous circadian system and by circadian misalignment in humans. International Journal of Obesity, 2019; 43: 1644-1649.

16. SANTANA CCA, et al. Physical fitness and academic performance in youth: A systematic review. Scandinavian Journal of Medicine \& Science in Sport, 2016; 27(6): 579-603.

17. SANTOS FS, et al. Estresse em Estudantes de Cursos Preparatórios e de Graduação em Medicina. Revista brasileira de educação médica [online], 2017; 41(2): 194-200.

18. SOCIEDADE BRASILEIRA DE PEDIATRIA, 2017. Manual de orientação - Promoção da atividade física na infância e na adolescência. Grupo de trabalho em atividade física.

19. TAJIRI E, et al. Effect of sleep curtailment on dietary behavior and physical activity: A randomized crossover trial. Physiology \& Behavior, 2018; 184: 60-67.

20. WORLD HEALTH ORGANIZATION, 2011. Global Recommendations on Physical Activity for Health 5-17 years old. 